Journal of Case Reports 2017;7(3):319-321

\title{
Skin Rash: An Early Clue to Systemic Vasculitis
}

\author{
Debabrata Mukherjee, Sourabh Sharma, Chilaka Rajesh, Ranjith K Nair, Bhaskar Datt \\ Department of Nephrology, Army Hospital Research \& Referral, Delhi.
}

\author{
Corresponding Author: \\ Dr. Sourabh Sharma \\ Email: drsourabh05@gmail.com \\ This is an Open Access article distributed \\ under the terms of the Creative Commons \\ Attribution License (creativecommons.org/ \\ licenses/by/3.0). \\ Received \\ Accepted \\ Published \\ May 1, 2017 \\ August 1, 2017 \\ September 25, 2017
}

\begin{abstract}
Background: Granulomatosis with polyangitis (GPA) is a distinct clinicopathological entity characterized by granulamatous vasculitis of upper and lower respiratory tract together with glomerulonephritis. Case Report: We present a case of GPA who presented with fever and skin rash on fifth day of fever. Skin biopsy revealed leucocytoclastic vasculitis and on further evaluation, he was found to be anti-neutrophil cytoplasmic antibody (ANCA) positive and renal biopsy was suggestive of ANCA associated vasculitis. Conclusion: It is important to be familiar with cutaneous manifestations of vasculitis as early diagnosis and prompt management is key to manage systemic vasculitic disorders.
\end{abstract}

Keywords: Antibodies, Biopsy, Exanthema, Glomerulonephritis, Vasculitis.

\section{Introduction}

Granulomatosis with polyangitis (GPA) is a distinct clinico-pathological entity characterized by granulamatous vasculitis of upper and lower respiratory tract together with glomerulonephritis. We present a case of GPA who presented with fever and arthralgia. He was managed initially as a viral fever but developed skin rash on fifth day of fever which helped in early diagnosis of his illness.

\section{Case Report}

A young well-built man with no previous comorbidities presented with joint pain which involved large joints. This was associated with intermittent high grade fever with chills and erythematous rash in both hands and feet [Fig.1]. On examination pallor and palpable purpuric rash was present. He was hemo-dynamically stable, ENT examination and systemic examination were unremarkable. Investigations revealed normochromic and normocytic anemia and persistent azotemia. Urine analysis showed active sediments and proteinuria. Imaging studies revealed nodular lesion in the lateral part of right middle lobe with central necrosis [Fig.2]. In view of renal, pulmonary and skin involvement a diagnosis of vasculitic disorder was considered.

On further evaluation he was found to be cytoplasmic anti-neutrophil cytoplasmic antibodies [c-ANCA (anti PR3)] positive (300 U/L). Skin biopsy [Fig.3a] showed dermal capillaries with fibrinoid necrosis, neutrophilic infiltration and karyorrhectic debris. Renal biopsy [Fig.3b] showed glomerulus with circumferential fibrocellular crescent and collapsed glomerular tuft in center. Mason trichome stain showed segmental collapse of glomerular tuft with fibrinoid necrosis.

Thus, final diagnosis of ANCA associated vasculitis was made and patient was successfully treated with intravenous cyclophosphamide along with methylprednisolone.

\section{Discussion}

GPA is ANCA associated small vessel vasculitis. Kumar A et al. found in their study that most cases present with upper or lower respiratory tract involvement $(84 \%)$, while skin manifestations constitute 32 percent [1]. Our patient presented initially with fever and arthralgia which responded 
to symptomatic treatment, later he developed skin rash which on biopsy showed leucocytoclastic vasculitis. On further investigations, patient was found to be c-ANCA and anti PR3 positive. Renal biopsy showed segmental collapse of glomerular tuft with fibrinoid necrosis and presence of fibrocellular cresents. Finally a diagnosis of ANCA associated vasculitis (granulomatosis with polyangitis) was made. Skin involvement is not unusual in GPA and occasionally may be a premonitory sign of the disease [2].

GPA requires both clinical and pathological features for diagnosis but not all patients demonstrate the classical triad (involvement of upper respiratory tract, lung and kidney). Furthermore, biopsy specimens from the lung and upper respiratory tract do not often show the classical pathologic triad of granulomatous inflammation, necrosis and vasculitis [3,4]. In GPA, although major histopathology changes are generally found in lung and upper respiratory tract biopsies (necrotizing granulomatous inflammation and leucocytoclastic vasculitis), they may also be depicted in cutaneous biopsies. So, in combination with findings from biopsy at usual sites, cutaneous findings could assist in early diagnosis and monitoring of the disease $[4,5]$.

Our patient was managed with intravenous methylprednisolone (1000 mg once daily for 3 days), intravenous cyclophosphamide (750 $\mathrm{mg}$ intravenous monthly) followed by tablet prednisolone $60 \mathrm{mg}$ with other supportive treatment with monthly monitoring of serum creatinine and urine sediment.

We reiterate through our case report that patients of GPA may not present with classic triad of upper and lower respiratory tract symptoms with kidney involvement. Skin rash should always be evaluated vigorously and early biopsy should be performed in relevant cases.

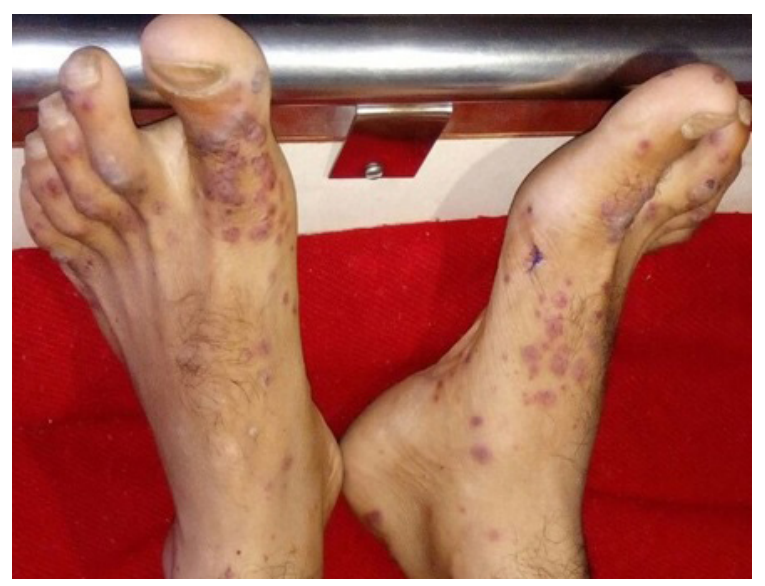

Fig.1: Bilateral peripheral maculopapular rash, which appeared on day 5.

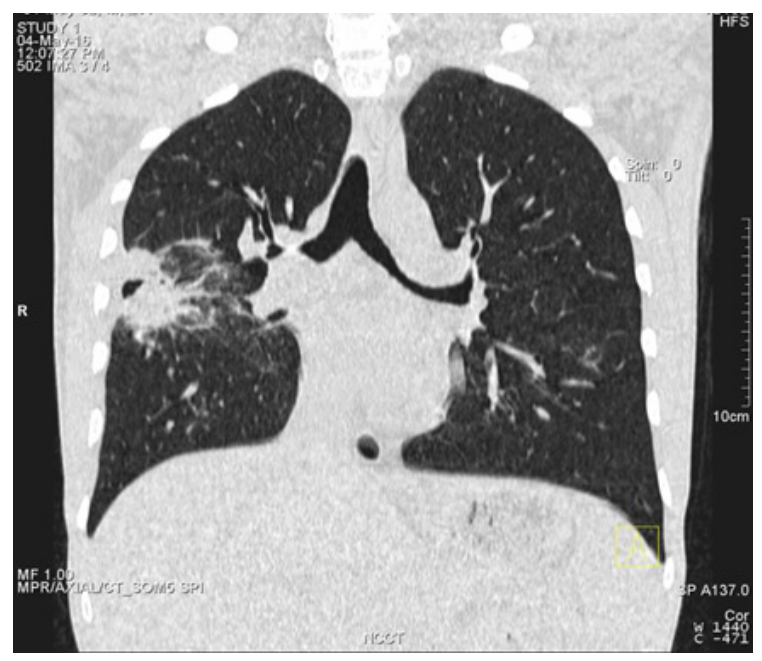

Fig.2: CT chest showing nodular lesion in the lateral part of right middle lobe with central necrosis.

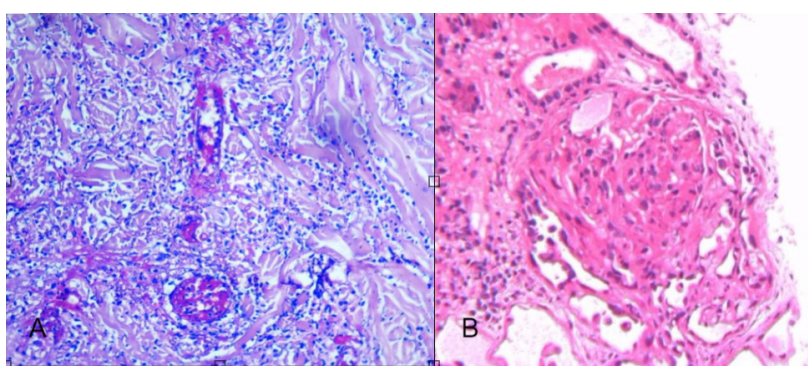

Fig.3(A): Skin biopsy (H\&E stain): showing dermal capillaries with fibrinoid necrosis, neutrophilic infiltration and karyorrhectic debris; (B): Kidney biopsy (H\&E stain): Glomerulus with circumferential fibrocellular crescent and collapsed glomerular tuft in center. 


\section{Conclusion}

It is important to be familiar with various cutaneous manifestations of vasculitis as early diagnosis and prompt management is a key to manage systemic vasculitic disorders.

Contributors: DM: manuscript editing, literature search, patient management; SS: manuscript writing, literature search, patient management; CR: manuscript editing, literature search; RKN, BD: critical inputs into the manuscript and patient management. SS will act as guarantor. All authors approved the final version of the manuscript.

Funding: None; Competing interests: None stated.

\section{References}

1. Kumar A, Pandhi A, Menon A, Sharma SK, Pande JN, Malaviya AN. Wegener's granulomatosis in India:
Clinical features, treatment and outcome of twenty five patients. Indian J Chest Dis Allied Sci. 2001;43:197-204.

2. Chhabra S, Minz RW, Rani L, Sharma N, Sakhuja $\mathrm{V}$, Sharma A. Immune deposits in cutaneous lesions of wegerner's granulomatosis: Predictor of an active disease. Indian J Dermatol. 2011;56:758-762.

3. Bambery P, Sakhuja V, Bhusnurmath SR, et al. Wegener's granulomatosis: clinical experience with eighteen patients. J Assoc Physicians India. 1992;40:597-600.

4. Handa R, Wali JP, Biswas A, Aggarwal P, Wig N. Wegener's granulomatosis: A clinicopathological study. J Assoc Physicians India. 1997;45:536-539.

5. Daoud MS, Gibson LE, DeRemee RA, Specks U, elAzhary RA, Su WP. Cutaneous Wegener's granulmatosis: clinical, histological and immune pathologic features of thirty patients. J Am Ac ad Dermatol. 1994;31:605-612. 